\title{
A chip-based silicon nitride platform for mid-infrared nonlinear photonics
}

\author{
Clemens Herkommer ${ }^{1,3}$, Hairun Guo ${ }^{1}$, Adrien Billat ${ }^{2}$, Davide Grassani ${ }^{2}$, Martin \\ Pfeiffer $^{1}$, Michael Zervas ${ }^{1}$, Camille-Sophie Brès ${ }^{2}$, Tobias J. Kippenberg ${ }^{1}$ \\ ${ }^{1}$ École Polytechnique Fédérale de Lausanne (EPFL), LPQM, CH-1015, Lausanne, Switzerland \\ ${ }^{2}$ École Polytechnique Fédérale de Lausanne (EPFL), PHOSL, CH-1015,Lausanne, Switzerland \\ ${ }^{3}$ Technische Universität München, Arcisstraße 21, D-80333 München Germany \\ Author email adress: ${ }^{1}$ clemens.herkommer@epfl.ch, ${ }^{3}$ clemens.herkommer@tum.de
}

\begin{abstract}
We developed a chip-based silicon nitride platform with thick waveguides $(>2 \mu \mathrm{m})$ that overcomes the usual fabrication limitation. We demonstrate both microresonator frequency comb generation at $2.5 \mu \mathrm{m}$ and supercontinuum generation extending beyond $4.0 \mu \mathrm{m}$ in this platform.
\end{abstract}

OCIS codes: 190.4390 (Nonlinear optics, integrated optics), 190.7110 (Ultrafast nonlinear optics)

Introduction. Chip-based silicon nitride $\left(\mathrm{Si}_{3} \mathrm{~N}_{4}\right)$ photonic waveguides represent an integrated platform compatible for both nonlinear photonics research and related applications [1], which benefits not only from advanced development of nanofabrication technology but also from the unique optical properties of $\mathrm{Si}_{3} \mathrm{~N}_{4}$ material. Indeed, the large bandgap of $\mathrm{Si}_{3} \mathrm{~N}_{4}$ supports a wide transparency from visible to mid-infrared (mid-IR), free of muli-photon absorptions. In addition, $\mathrm{Si}_{3} \mathrm{~N}_{4}$ has strong nonlinear efficiency (10 times of silica) that provides rich nonlinear phenomena within chip-scale devices. A vivid example are chip-based microresonator frequency combs that feature fully coherent optical spectra with large repetition rate $(>10 \mathrm{GHz})$ and broad bandwidth $[2,3]$. Such comb sources have been used in novel applications such as coherent telecommunication [4], dual-comb spectroscopy [5] and low-noise microwave generation [6,7]. However, despite the ultra-broad transparency, $\mathrm{Si}_{3} \mathrm{~N}_{4}$ photonic diveces to date are mainly working in the telecommunication band. Limitations for the mid-IR include the technical challenge that larger waveguides can possibly lead to cracks, as well as limited flexibility, e.g. in terms of dispersion engineering. On the other hand, a mid-IR chip-based platform is desired particularly for spectroscopy applications.

Here we developed a $\mathrm{Si}_{3} \mathrm{~N}_{4}$ platform with thick waveguides $(>2 \mu \mathrm{m})$ beyond the limitation of conventional fabrication, allowing for mid-IR photonics research. As proof of concept we demonstrate both microresonator frequency comb generation (at $2.5 \mu \mathrm{m}$ ) and supercontinuum generation (extending to $4.0 \mu \mathrm{m}$ ) in these devices.
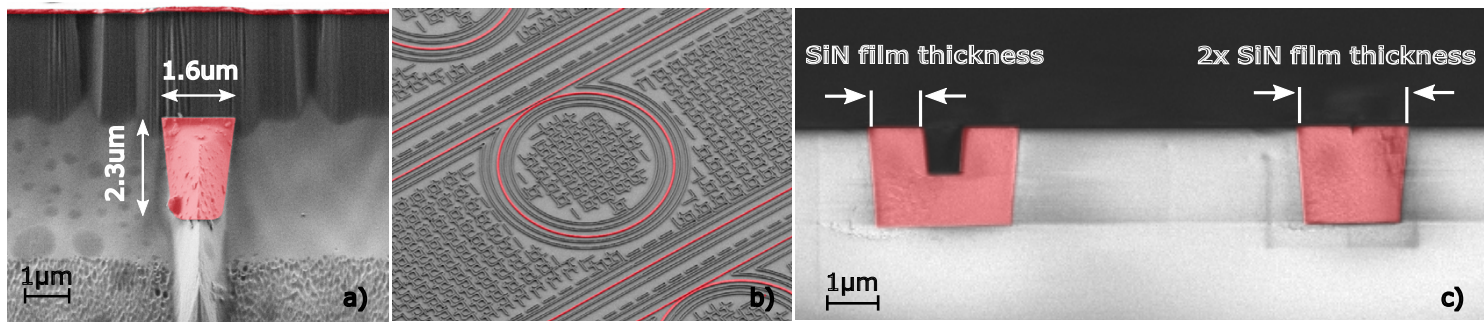

Fig. 1. a) Cross section of a fabricated waveguide showing the large core dimensions. b) SEM image of a microresonator without the $\mathrm{SiO}_{2}$ cladding. c) SEM image of a cross section of waveguides after planarization. Trenches with a width below the threshold of 2 times the deposition thickness (right-hand side) are filled completely with SiN due to the isotropic deposition process.

Fabrication. We fabricate the devices using a modified Damascene Process that was recently reported [8] as an approach to reliably fabricate crack-free waveguides. Important advantages are the possibility to employ a "Reflow" step that yields highly smooth sidewalls, as well as 3D-tapered bus waveguide ends that enlarge the mode size and facilitate input coupling. Different from subtractive fabrication processes, here $\mathrm{Si}_{3} \mathrm{~N}_{4}$ is deposited onto a structured $\mathrm{SiO}_{2}$ preform, where a dense filler pattern is employed to prevent crack formation originating from the tensile stress of LPCVD-deposited $\mathrm{Si}_{3} \mathrm{~N}_{4}$.

In order to achieve crack-free $\mathrm{Si}_{3} \mathrm{~N}_{4}$ waveguides with a large core-size geometry, in the fabrication process we circumvent the crack-prone increase of the $\mathrm{Si}_{3} \mathrm{~N}_{4}$ film thickness by making use of the isotropic deposition of $\mathrm{SiN}$ during LPCVD, as can be seen in figure 1. In this way we can freely choose the waveguide height by merely adjusting the etching time, whereas the width of the waveguide is limited to be less than twice the deposited $\mathrm{Si}_{3} \mathrm{~N}_{4}$ film thickness. 
a)

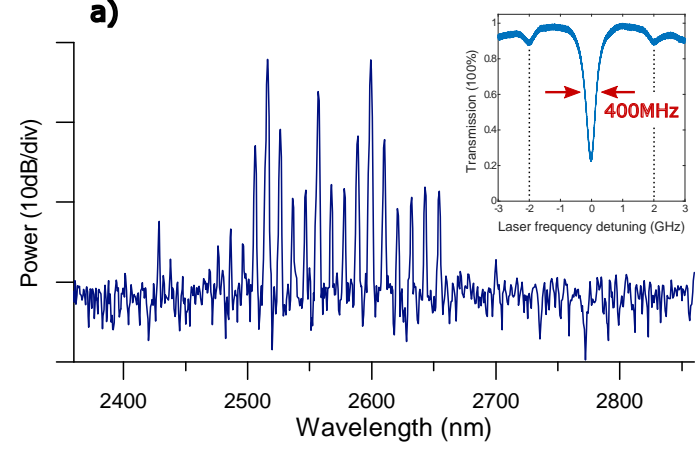

b)

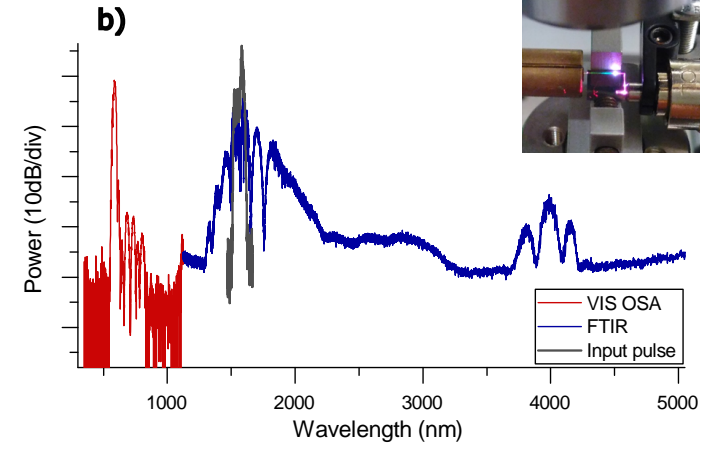

Fig. 2. a) Mid-IR Kerr comb spectrum generated in a 500-GHz Si $\mathrm{N}_{4}$ microresonator with an intrinsic $Q$ of 600,000 , pumped at $2.55 \mu$ m. The inset shows a measured resonance calibrated by EOM-induced 2-GH sidebands. b) Supercontinuum generation in a 5-mm $\mathrm{Si}_{3} \mathrm{~N}_{4}$ waveguide, pumped at $1.55 \mu \mathrm{m}$. Mid-IR dispersive waves are up to $4.0 \mu \mathrm{m}$. Strong visible dispersive waves are also observed

With this approach we are able to fabricate waveguides with a width of $1.6 \mu \mathrm{m}$ and a height of $2.3 \mu \mathrm{m}$, with negligible sidewall roughness as enabled by the reflow step on the $\mathrm{SiO}_{2}$ preform.

Microresonator frequency comb generation. We fabricated $\mathrm{Si}_{3} \mathrm{~N}_{4}$ microresonators with a waveguide geometry that is designed to produce anomalous dispersion at $2.5 \mu \mathrm{m}$ in order to support Kerr frequency comb generation when pumping with a continuous-wave laser. We measured the resonance linewidth of $\sim 400 \mathrm{MHz}$ corresponding to an intrinsic $Q$-factor of 600,000 in resonators with a free spectral range (FSR) of $200 \mathrm{GHz}$ at $2.55 \mu \mathrm{m}$ by using a freespace CW optical parametric oscillator (CW-OPO) seeded by an external cavity diode laser (ECDL) at $1.064 \mu \mathrm{m}$. For comb generation from these resonators, the power threshold of modulation instability is estimated as $\sim 250 \mathrm{~mW}$. In resonators with a 500-GHz FSR, the threshold is reduced to be below $100 \mathrm{~mW}$, though the resonance linewidth is also slightly degraded. In experiments we employed the laser tuning method to generate the Kerr comb [9]. Figure 2a) shows the spectrum of a frequency comb generated in a 500-GHz microresonator where the first side band appeared at $4 \times$ FSR from the pump. We notice this comb is still in the chaotic state in the operation regime of modulation instability. The transition to the low-noise state with the formation of dissipative Kerr solitons was not observed, most likely as the system is short on the pump power.

Supercontinuum generation. With the same process we also fabricated $\mathrm{Si}_{3} \mathrm{~N}_{4}$ waveguides that support supercontinuum generation. We pumped the waveguides with a femtosecond pulsed laser at $1.55 \mu \mathrm{m}$ with pulse duration 130 fs and pulse energy $0.6 \mathrm{~nJ}$. Figure $2 \mathrm{~b}$ ) shows the spectrum of a supercontinuum generated in a $\mathrm{Si}_{3} \mathrm{~N}_{4}$ waveguide with a length as short as $5 \mathrm{~mm}$. A typical spectral broadening regime was observed: at the pump wavelength, the spectral broadening is dominated by self-phase modulation of the seeded pulse as the soliton number is estimated to be larger than unity, indicating soliton self-compression. Interestingly, we observed strong dispersive wave packets in both the visible $(\sim 650 \mathrm{~nm})$ and mid-IR $(4.0 \mu \mathrm{m})$ range, caused by soliton induced Cherenkov radiation. Backing on the dispersive waves, the overall span of the supercontinuum is larger than two octaves. Remarkably, the $\mathrm{Si}_{3} \mathrm{~N}_{4}$ waveguide provides a decent confinement of mid-IR waves thanks to the large geometry.

Discussion. We demonstrated the capability of the $\mathrm{Si}_{3} \mathrm{~N}_{4}$ platform in fabricating thick waveguides that provide access to mid-IR photonics including both microresonator frequency comb generation and supercontinuum generation. This work represents a solid first step on the route towards generating mid-IR soliton-based coherent microresonator frequency combs for chip-based spectroscopy devices.

Acknowledgements: This publication was supported by contract W31P4Q-16-1-0002 (SCOUT) from the Defense Advanced Research Projects Agency (DARPA), Defense Sciences Office (DSO). This material is based upon work supported by the Air Force Office of Scientific Research, Air Force Material Command, USAF under Award No. FA9550-15-1-0099. AB, DG and CSB acknowledge support from the European Research Council under grant agreement ERC-2012- StG 306630-MATISSE.

\section{References}

1. J. Levy et al., "CMOS-compatible multiple-wavelength oscillator for on-chip optical interconnects," Nature Photon. 4, 37-40 (2010).

2. V. Brasch et al., "Photonic chip-based optical frequency comb using soliton Cherenkov radiation," Science 351, 357-360 (2016).

3. M. Karpov et al., "Raman self-frequency shift of dissipative Kerr solitons in an optical microresonator," Phys. Rev. Lett. 116, 103902 (2016)

4. P. Marin-Palomo et al., "Microresonator solitons for massively parallel coherent optical communications," arXiv: 1610.01484 (2016).

5. M.-G. Suh et al., "Microresonator soliton dual-comb spectroscopy," Science 354, 600-603 (2016).

6. J. Jost et al., "Counting the cycles of light using a self-referenced optical microresonator," Optica 2, 706-711 (2015).

7. V. Brasch et al., "Self-referencing of an on-chip soliton Kerr frequency comb without external broadening," arXiv: 1605.02801 (2016).

8. M.H.P. Pfeiffer et al., "Photonic Damascene process for integrated high- $Q$ microresonator based nonlinear photonics", Optica 3, 20-25 (2016).

9. T. Herr et al., "Universal formation dynamics and noise of Kerr-frequency combs in microresonators" Nature photonics 6, 480-487 (2012). 